\section{Lead identification and screening}

Amphora Discovery

ArQule

Astex Technology

Chemical Abstracts Service

Chembridge

Chemical Diversity Labs

Chemspeed

CombinatoRx

CombiPure

De Novo Pharmaceuticals

Evotec OAl

Graffinity

Iconix

MDL

MDS Pharma

Plexxikon

Schrödinger

Serenex

Signal Pharmaceuticals

Tripos

Chemical library services
Lead discovery and screening

Chemical synthesis, lead identification

Fragment-based drug discovery, computational screening, high-throughput X-ray crystallography, software for protein-ligand structure determination

SciFinder chemical information database

Small-molecule libraries for screening

Target collection for lead discovery, target-specific libraries, building-block libraries

Automated chemical synthesis workstations, peptide synthesizers

Lead compound identification and screening; drug discovery

In silico drug-design technology

Services for drug discovery: compound libraries, target assays, medicinal chemistry

Drug-discovery using chemical microarrays, imaging of protein-ligand interactions

DrugMatrix databases and software for chemogenomics-based research

CrossFire Beilstein, CrossFire Gmelin and other chemistry-related databases

Services company for all aspects of drug discovery

Proprietary scaffold-based Drug Discovery platform for small-molecule inhibitors

Molecular modelling for in silico screening

Screening against proteins; functional proteome fractionation; proteome mining

Target and lead discovery in signalling pathways

Chemical libraries; modelling, pharmacophore perception and screening software
North Carolina

Woburn, Massachusetts

Cambridge, UK

Columbus, Ohio

San Diego, California

San Diego, California

Augst, Switzerland

Boston, Massachusetts

Kegworth, UK

Cambridge, UK

Hamburg, Germany

Heidelberg, Germany

Mountain View, California

San Leandro, California

Lincoln, Nebraska

Berkeley, California

Portland, Oregon

Durham, North Carolina

San Diego, California

St Louis, Missouri www.amphoracorp.com

www.arqule.com

www.astex-technology.com

\section{www.cas.org}

www.chembridge.com

www.chemdiv.com

www.chemspeed.com

www.combinatorx.com

www.combipure.com

www.denovopharma.com

www.evotecoai.com

www.graffinity.com

www.iconixpharm.com

www.beilstein.com

www.mdsps.com

www.plexxikon.com

www.schrodinger.com

www.serenex.com

www.signalpharm.com

www.tripos.com
Cell-based assay systems and high-content screening

Aclara BioSciences

Asys Hitech

Axon Instruments

Beckman Coulter

Biomol

\section{Bio-Tek}

BMG Labtechnologies

Caliper Life Sciences

Carl Zeiss Jena

Cellectricon

Cellomics

CyBio

Cytogration

DiscoveRx

Dynex Technologies

EUGENEX Biotechnologies

GE Healthcare Bio-Sciences

Jerini Array Technology

KeyNeurotek

Molecular Devices

OptiCell

PerkinEImer Life Sciences

Protedyne

QIAGEN

RTS Life Sciences

SEPIAtec

Tecan

TTP LabTech

Velocity 11

Vitra Biosciences

Wave Biotech

Xcellsyz

Microplate readers, washers and dispensers

ImageExpress high-throughput system for live-cell assays

SAGIAN core system for high-content screening

Reagents for signal transduction research

Microplate and array readers and handling systems

LabChip microfluidic systems for high-throughput screening

Patch-clamp equipment for ion-channel screening

Cell-based assay development

Microplate readers, washers and automated workstations

Development of test cell lines

Functionality and tissue-based screening systems for integration into robotic systems

Automated cell-culture devices

research; confocal laser scanning microscope for live-cell imaging

BioCube automated systems acid, protein and cell purification

Automated compound storage/retrieval systems

Immortalized cell lines, cell-based assays, screening
LabCard 'lab-on-a-chip' systems for biochemical and cellular analysis

Automated microplate handling and reading instrumentation, spectrophotometers

plate::explorer and plate::screen systems for ultra-high-throughput screening

ArrayScan HCS and KineticScan HCS systems for automated cell-based screening

Pipetting, liquid handling, incubation and imaging systems for automated screening

Automated high-throughput cell culture systems for in vitro screening

Instruments, equipment and products for life-sciences research; LEADseeker for lead

identification, IN Cell Analyzer confocal imaging system for rapid cellular assays

Substrate identification for kinases using pepSTAR peptide microarray technology

Mountain View, California

Eugendorf, Austria

Foster City, California

Fullerton, California

Plymouth Meeting,

Pennsylvania

Winooski, Vermont

Offenburg, Germany

Hopkinton, Massachusetts

Göttingen, Germany

Gothenburg, Sweden

Pittsburgh, Pennsylvania

Jena, Germany

Rockville, Maryland

Fremont, California

Chantilly, Virginia

Taegerwilen, Switzerland

Little Chalfont, UK

Berlin, Germany

Magdeburg, Germany

Liquid-handling and microplate-processing equipment, imaging instruments and assay Sunnyvale, California

Westerville, Ohio

Automated systems for liquid handling and sample preparation for drug discovery and Boston, Massachusetts

BioRobot multifunctional lab workstation and equipment for automated nucleic

Automated sample management, liquid handling, ultra-high-throughput screening

HPLC/SPE equipment for high-throughput purification and fractionation

Laboratory automation, LabCD 'lab-on-a-disc' assays; Cellerity cell-culture system

Automated high-throughput microplate processing, dispensers and pipetting stations

CellCards and CellPlex system for simultaneous assays of multiple cell types

Equipment for cell culture: Wave Bioreactor disposable cell-culture systems

\section{Windsor, Connecticut}

Valencia, California

Manchester, UK

Berlin, Germany

Männedorf, Switzerland

Royston, UK

Palo Alto, California

Mountain View, California

Bridgewater, New Jersey

Newcastle upon Tyne, UK www.aclara.com

www.asyshitech.com

www.axon.com

www.beckmancoulter.com

www.biomol.com

www.biotek.com

www.bmg-labtechnologies.com

www.calipertech.com

www.zeiss.com

www.cellectricon.se

www.cellomics.com

www.cybio-ag.com

www.cytogration.com

www.discoverx.com

www.dynextechnologies.com

www.eugenex.com

www.amersham.co.uk

www.jerini.com

www.keyneurotek.de

www.moleculardevices.com

www.opticell.com

lifesciences.perkinelmer.com

www.protedyne.com

www.qiagen.com

www.rts-group.com

www.sepiatec.com

www.tecan.com

www.ttplabtech.com

www.velocity11.com

www.vitrabio.com

www.wavebiotech.com

www.xcellsyz.com

\section{General}

Affymetrix

Agilent

Analytikjena

Apogent Technologies

Applied Biosystems
DNA microarrays; arrayers; imaging systems and software

Santa Clara, California 2100 Bioanalyzer for RNA, DNA and proteins; instruments for genomics and proteomics Palo Alto, California Instrumentation for molecular spectroscopy, affinity measurements, and plate readers Labware and equipment for life sciences

DNA and protein sequencers; mass spectrometers; chromatography and PCR analysis; peptide and nucleic-acid synthesizers; ICAT stable isotope labelling reagents
Jena, Germany

Portsmouth, New Hampshire

Foster City California www.affymetrix.com

www.agilent.com

www.analytikjena.com

www.apogent.com

home.appliedbiosystems.com 


\begin{tabular}{|c|c|c|c|}
\hline Company & Products/activity & Location & URL \\
\hline Applied Scientific Instrumentation & Automated systems for microscopy and fluorescence screening & Eugene, Oregon & www.asiimaging.com \\
\hline BD Biosciences & Culture media, radioassay kits, FACS flow cytometers, monoclonal antibodies & Franklin Lakes, New Jersey & www.bd.com \\
\hline Berthold Technologies & Bioanalytical instrumentation & Bad Wildbad, Germany & www.bertholdtech.com \\
\hline Bibby Sterilin & Laboratory glassware, consumables and equipment for life-sciences research & Stone, UK & www.bibby-sterilin.co.uk \\
\hline Biophile & Ultra-low-temperature sample storage and retrieval system & Charlottesville, Virginia & www.biophileinc.com \\
\hline Bio-Rad & Products, instruments and software for life-sciences research & Hercules, California & www.bio-rad.com \\
\hline Bioventures & Products and services for genomics and proteomics & Murfreesboro, Tennessee & www.bioventures.com \\
\hline Brandel & Sample preparation and harvesting, superfusion systems and microdispensers & Gaithersburg, Maryland & www.brandel.com \\
\hline Bruker & Automated platforms for mass spectrometry, NMR, EPR and MRI & The Woodlands, Texas & www.bruker.com \\
\hline Chromagen & Fluorescence-based products, kits and services for protein detection and assay & San Diego, California & www.chromagen.com \\
\hline Corning & Labware and laboratory equipment for life sciences & Corning, New York & www.corning.com \\
\hline Deerac Fluidics & Nanovolume pipetting systems & Dublin, Ireland & www.allegro-technologies.com \\
\hline EMD Biosciences & Novabiochem, Calbiochem, Novagen and Oncogene Research products & San Diego, California & splash.emdbiosciences.com \\
\hline Gilson & Automated liquid-handling, pipetting and protein-crystallization instruments & Middleton, Wisconsin & www.gilson.com \\
\hline Greiner Bio-One & Consumables for high-throughput research and diagnostics; microplates & Frickenhausen, Germany & www.gbo.com/bioscience \\
\hline Hamamatsu & Imaging and analysis systems & Hamamatsu City, Japan & www.hamamatsu.com \\
\hline Hamilton Company & Microlab automated liquid-handling workstations & Reno, Nevada & hamiltoncomp.com \\
\hline Hitachi High-Technologies & $\begin{array}{l}\text { Automated instrumentation for mass spectroscopy, liquid chromatography, } \\
\text { high-throughput purification, amino-acid analysis and spectroscopy }\end{array}$ & Tokyo, Japan & www.hitachi-hitec.com \\
\hline IBM Life Sciences & DiscoveryLink platform for database integration; data-management systems & White Plains, New York & $\begin{array}{l}\text { www-1.ibm.com/industries/ } \\
\text { lifesciences }\end{array}$ \\
\hline Integra Biosciences & Equipment/consumables for sterilization, liquid handling, cell culture, sample storage & Baar, Switzerland & www.integra-biosciences.com \\
\hline Invitrogen & Kits and reagents for cloning, genomics, proteomics, molecular and cell biology & Carlsbad, California & www.invitrogen.com \\
\hline KBiosystems & Robotic instrumentation for high-throughput genomics and proteomics & Basildon, UK & www.kbiosystems.com \\
\hline Kimble-Kontes & Laboratory glassware & Vineland, New Jersey & www.kimble-kontes.com \\
\hline L.I.M.S. & StarLIMS laboratory information management system & South Hollywood, Florida & www.starlims.com \\
\hline PhyNexus & Single-use pipette-tip-based chromatography columns for protein purification & San Jose, California & www.phynexus.com \\
\hline Pierce Chemical & Components and consumables for automated systems in the life sciences & Rockford, Illinois & www.piercenet.com \\
\hline PreAnalytiX & Products and reagents for nucleic-acid isolation & Hombrechtikon, Switzerland & www.preanalytix.com \\
\hline Promega & Reagents and kits for genomics, proteomics and cellular analysis & Madison, Wisconsin & www.promega.com \\
\hline Robbins Scientific & Labware, instruments and automated liquid-handling and dispensing equipment & Sunnyvale, California & www.robsci.com \\
\hline RoboDesign & Automated equipment for biotechnology & Carlsbad, California & www.robodesign.com \\
\hline Roche Diagnostics & Reagents and kits for molecular biology & Lewes, UK & www.roche-applied-science.com \\
\hline Rohasys & Automated equipment for sample pretreatment, weighing, pH, conductivity, turbidity & Rijen, the Netherlands & www.rohasys.com \\
\hline Sankyo Robotics & Multipurpose robots & Boca Raton, Florida & www.sankyo.com/robotics \\
\hline Scinomix & Robotics and laboratory LIMS software for biotechnology & Earth City, Missouri & www.scinomix.com \\
\hline Shimadzu Biotech & AXIMA MALDI QIT time-of-flight mass spectrometer for protein identification & Kyoto, Japan & www.shimadzu-biotech.net \\
\hline Sirius & Instrumentation and analysis software for pKA, pH and membrane permeability & Forest Row, UK & www.sirius-analytical.com \\
\hline Stratagene & Tools and reagents for molecular biology, genomics, proteomics, drug discovery & La Jolla, California & www.stratagene.com \\
\hline Takara Bio & $\begin{array}{l}\text { Reagents, kits and custom services for molecular biology, genomics, proteomics } \\
\text { research; PCR enzymes; DNA microarrays; protein-refolding kit }\end{array}$ & Shiga, Japan & www.takara-bio.co.jp/english \\
\hline TekCel & Low-temperature compound storage and retrieval & Hopkinton, Massachusetts & www.tekcel.com \\
\hline The Automation Partnership & Automation for liquid handling, compound storage, high-throughput screening & Royston, UK & www.automationpartnership.com \\
\hline Thermo Electron & Spectrometry, chromatography, microscopy, liquid handling, robotics & Waltham, Massachusetts & www.thermo.com \\
\hline Tomtec & Liquid-handling workstations, solid-phase extraction workstations & Hamden, Connecticut & www.tomtec.com \\
\hline Torcon Instruments & Precision fluid dispensing and automated instrumentation for microplate handling & Torrance, California & www.torconinstruments.com \\
\hline Universal Technology & Automated storage and retrieval systems for the pharmaceutical industry & Pittsburgh, Pennsylvania & www.univtech.com \\
\hline Upstate & $\begin{array}{l}\text { KinaseProfiler specificity testing, IC50Profiler Express, crystallography assays, } \\
\text { bulk protein production, chromatin immunoprecipitation kits, signalling proteins }\end{array}$ & Charlottesville, Virginia & www.upstate.com \\
\hline Walden Precision Apparatus & pH meters, conductivity meters, colorimeters, spectrophotometers, oxygen meters & Cambridge, UK & www.wpaltd.co.uk \\
\hline Warner Instruments & Electrophysiology and cell-biology equipment and products & Hamden, Connecticut & www.warneronline.com \\
\hline Waters & Micromass instruments for mass spectroscopy; HPLC systems & Milford, Massachusetts & www.waters.com \\
\hline
\end{tabular}

- see advertisement 